${ }^{2}$ Department of Rheumatology, CEDAR Superspeciality Clinics, Dwarka, New Delhi, India

${ }^{3}$ Department of Cardiology, Janakpuri Superspeciality Hospital, New Delhi, India

${ }^{4}$ Department of Endocrinology, Apex Hospitals, Rohtak, India

${ }^{5}$ Department of Endocrinology, Maharaj Agrasen Hospital, Punjabi Bagh, New Delhi, India

\title{
Glycaemic and weight-loss outcomes of graded doses of canagliflozin in type 2 diabetes - a real-world study
}

\section{ABSTRACT}

Background. Costs are the most important cause of therapeutic non-compliance. Half canagliflozin (CANA)-300 tablet has lowest cost/mg among all CANA preparations; data are unavailable on efficacy of half CANA-300. This study evaluated weight loss and glycaemic outcomes of $100 \mathrm{mg}$ versus $150 \mathrm{mg}$ versus $300 \mathrm{mg}$ of canagliflozin as part of standard therapy. Methods. Data, retrospectively captured from medical records of two centres in Delhi for patients $>\mathbf{3 5}$ years with type-2 diabetes (T2DM), and on canagliflozin, having $>6$ months follow-up, were analysed. Patients were in 3-groups depending on canagliflozin dosage: Group 1 on canagliflozin 100 mg/day (1 tablet CANA-100), Group-2 on canagliflozin $150 \mathrm{mg} /$ day (half tablet CANA-300), and Group 3 on canagliflozin $300 \mathrm{mg} / \mathrm{day}$ (1 tablet CANA-300). Primary endpoints were glycaemic efficacy and weight-loss.

Results. From 3,569 records evaluated, 1,232 people with T2DM on canagliflozin were screened; data from 528 individuals analysed (257, 138 and 133 in Groups: 1, 2 and 3 respectively). People in all three groups were comparable with regards to sex, T2DM

Address for correspondence:

Deep Dutta, Department of Endocrinology,

Center for Endocrinology, Diabetes,

Arthritis \& Rheumatism (CEDAR) Superspecialty Center,

Plot 107 \&108 Block-A, Sector-12,

Dwarka, New Delhi, India 110075

e-mail: deepdutta2000@yahoo.com

Clinical Diabetology 2020, 9; 6: 442-453

DOI: $10.5603 /$ DK.2020.0044

Received: 12.08 .2020

Accepted: 12.09 .2020 duration, glycated haemoglobin $\left(\mathrm{HbA}_{1 \mathrm{c}}\right)$, haemoglobin, creatinine, lipids, albuminuria and medications. Group-2 patients were youngest and had highest BMI. Following 6-months, both absolute and percent weight-loss was significantly higher in Group-2 $(-3.5 \mathrm{~kg}$ [-6.60-0.00]; $-3.62 \%)$, followed by Group-3 $(-3.0 \mathrm{~kg}$ [-5.3 to -0.8$] ;-3.33 \%)$, and lowest in Group-1 $(-1.05 \mathrm{~kg}$ [-2.85 to -0.17$] ;-1.31 \%)(P=0.002$ and 0.014 , respectively). Glycaemic efficacy was comparable among groups.

Conclusion. Half CANA-300 tablet has comparable glycaemic efficacy and weight-loss compared to single CANA-300 tablet, but superior weight-loss compared to CANA-100. (Clin Diabetol 2020; 9; 6: 442-453)

Key words: obesity canagliflozin, weight loss, diabetes reversal, euglycaemia, type 2 diabetes, cost analysis

\section{Introduction}

Diabetes and obesity, or diabesity, has become a global pandemic. Recent studies have suggested an alarming burden of diabetes and obesity in the Indian population. India currently has an overall $9 \%$ and $14-18 \%$ prevalence of diabetes and prediabetes, respectively [1, 2]. Indians are metabolically challenged, as is evidenced by nearly two decades earlier onset of diabetes coupled with one of the highest global annual rates of prediabetes progression to diabetes (14.0-18.0\%, $11.0 \%, 6.0 \%$ and $2.5 \%$ per annum in India, China, Finland and USA, respectively) [3]. The 
problem is especially acute in urban areas. The CARRS study, a population screening of 5,365 individuals from New Delhi, revealed a very high prevalence of prediabetes/diabetes of $72.7 \%$ [4]. Two studies from New Delhi reported very high rates of obesity in the general population $(71.50 \%$ and $69.29 \%$ in a cohort of 1,473 and 5,336 patients, respectively) $[5,6]$. Hence, it is the high prevalence of obesity which is driving this diabetes epidemic, especially in the urban areas.

Recent studies have demonstrated the importance of weight loss in not only ensuring better glycaemic control in type 2 diabetes mellitus (T2DM), but also in diabetes remission [7]. Sodium-glucose co-transporter-2 (SGLT2) inhibitors have become popular agents for managing diabetes especially in the setting of diabesity. This is due to their good glycaemic efficacy, glycaemic durability, beneficial impact on cardiovascular outcomes, low risk of hypoglycaemia, along with their mild weight-loss properties [8].

There are currently four SGLT2 inhibitors available for clinical practice in India: canagliflozin, dapagliflozin, empagliflozin and remogliflozin. In terms of selectivity for the inhibition of SGLT2, SGLT1 transporter, empagliflozin, is the most selective, whereas canagliflozin is the least selective [8]. Hence canagliflozin is believed to have some additional therapeutic potential in view of its inhibiting SGLT1 transporters present in the intestines [9]. No significant impact of canagliflozin on SGLT1 transporter in heart and kidneys has been documented [10]. The intestinal SGLT1 transporter inhibition by canagliflozin is believed to result in an additional post-prandial glucose reduction, which is not seen with other SGLT2 inhibitors [10].

One of the major limitations with long-term use of SGLT2 inhibitors in clinical practice, is the significantly increased monthly costs of treatment. Since most of the healthcare expenditure is out of pocket in India, increased monthly treatment costs have been linked to poor medication compliance, resulting in impaired glycaemic control [11, 12]. Canagliflozin is currently available in $100 \mathrm{mg}$ and $300 \mathrm{mg}$ tablets for clinical use in India, costing INR 54.5 (INR 0.55/mg; INR 1,635 per month) and INR 120 (INR 0.4/mg; INR 3,600 per month) per tablet, respectively $[13,14]$. Treatments costs for sulfonylurea glipizide $5 \mathrm{mg}$, metformin sustained release preparation $1 \mathrm{~g}$, and pioglitazone $15 \mathrm{mg}$ are: INR 0.53 per tablet (monthly cost INR 63.6 for $20 \mathrm{mg}$ therapy per day), INR 3 per tablet (monthly cost INR 180 for $2 \mathrm{~g}$ therapy per day) and INR 2 per tablet (monthly cost INR 120 for $30 \mathrm{mg}$ therapy per day), respectively, highlighting nearly 10-60-times increased cost burden with SGLT2 inhibitor use in India [15]. The cost per unit of human regular insulin, human neutral protamine
Hagedorn (NPH) insulin, lispro insulin, aspart insulin and glargine insulin is INR 0.96, 0.96, 2.26 and 2.19, respectively, when used in the form of cartridges for pen fill [16-19]. The monthly cost of therapy of above 4 insulins when taken at doses of $20 \mathrm{U} / \mathrm{d}$ would be INR 580, 580, 1,356 and 1,314 respectively [16-19]. Hence monthly costs of human insulin analogous (both short- and long-acting) is almost similar to that of SGLT2 inhibitors.

From costing point of view, half tablet of canagliflozin $300 \mathrm{mg}$ (CANA-300) would provide $150 \mathrm{mg}$ of canagliflozin at INR 60, which would be much more cost effective than taking 1.5 tablets of canagliflozin $100 \mathrm{mg}$ (CANA-100) tablet at INR 81.75. However, no data are available on the glycaemic and weight-loss properties of half tablet of CANA-300 taken once a day as compared to 1 tablet of CANA-100 per day and 1 tablet of CANA-300 per day in clinical practice. Half CANA-300 tablet has the lowest cost per mg as well as the lowest monthly cost of therapy among all the different doses of canagliflozin available for clinical use. Hence, this study aimed to evaluate the glycaemic efficacy and weight loss properties of graded doses of canagliflozin (100 mg, $150 \mathrm{mg}, 300 \mathrm{mg}$ ), as a part of multi-drug therapy for managing type- 2 diabetes in India.

\section{Methods}

Data were retrospectively captured from the electronic medical record (EMR) database of two different centres in New Delhi. Patients with T2DM, aged > 35 years and on canagliflozin were considered for the study. T2DM onset in Indians is nearly 2 decades earlier than the western world, and the peak age of T2DM onset in Indians is in 30s and 40s [1, 20]. People > 35 years-age were considered for this study to rule out those who were likely to have latent onset autoimmune diabetes of adults and late onset T1DM [1, 20].

Patients with associated severe chronic co-morbid states like chronic liver disease (Child's B or C), renal disease (glomerular filtration rate $<60 \mathrm{~mL} / \mathrm{min}$ as calculated by CKD-EPI formula), cardiac disease (including coronary artery disease and heart failure), malignancies, active infection (tuberculosis, HIV, viral hepatitis), post organ transplant, patients on psychiatry medications, and those with chronic autoimmune disorders (lupus, scleroderma), were excluded. Also, patients with history of hospital admission in the last 6 months were excluded [6]. Patients with prior use of SGLT2 inhibitors were excluded. Incomplete records were excluded from the analysis. Details of other medications being used as per standard care were noted [6]. Patients on any other medications which can cause weight loss apart 


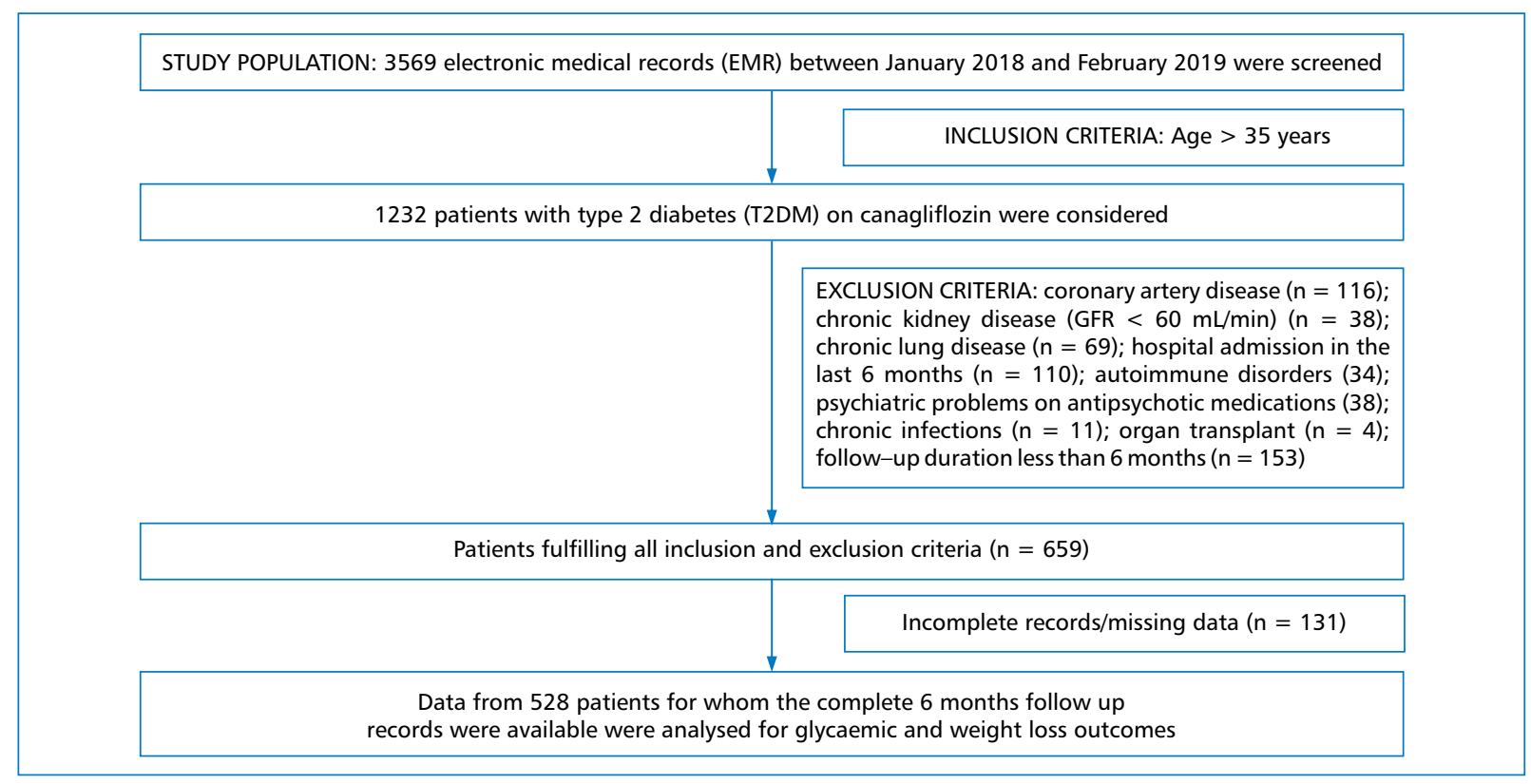

Figure 1. Flowchart of study protocol and flow of patients. GFR - glomerular filtration rate

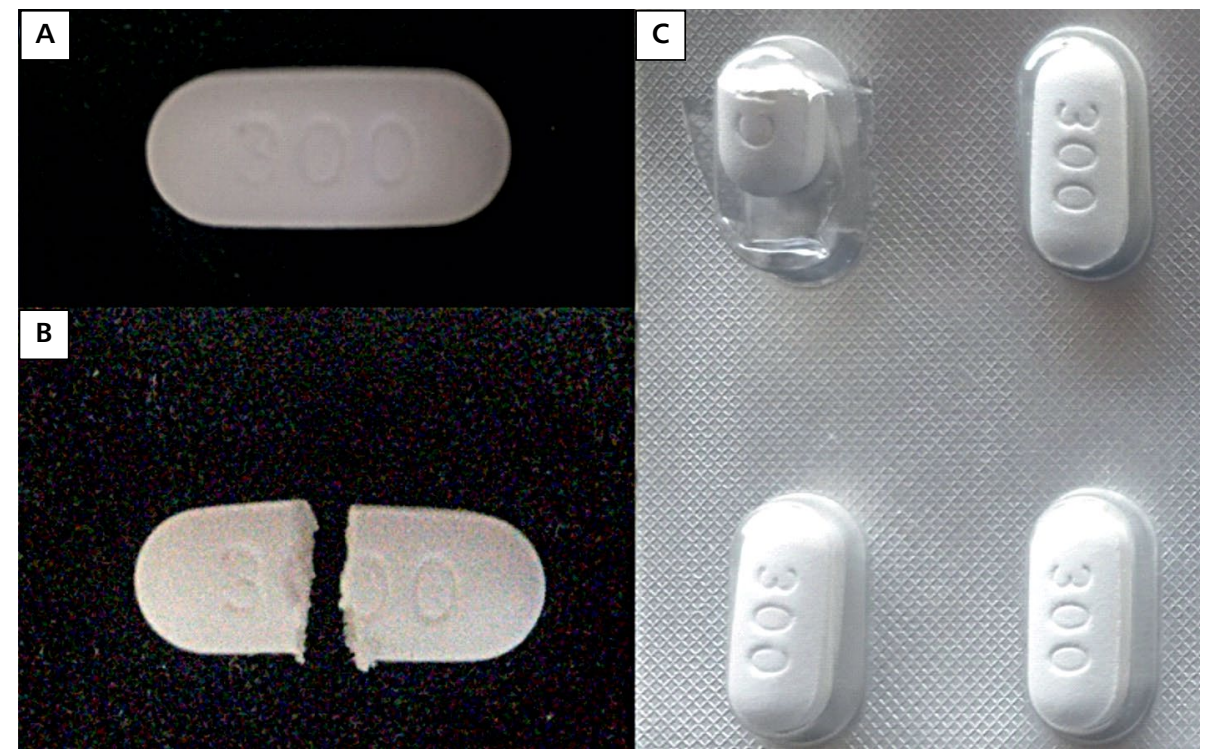

Figure 2. A - appearance of an intact canagliflozin $300 \mathrm{mg}$ tablet; B - appearance of split canagliflozin $300 \mathrm{mg}$ tablet; C - storage of one-half of the split canagliflozin $300 \mathrm{mg}$ tablet of use on the subsequent day

from the medications considered in this study (canagliflozin, metformin, glucagon like peptide 1 receptor agonists and orlistat) were excluded. Patients with at least 6 months follow-up data available were included in the study. The duration of this study was from January 2018 to February 2019. The entire flow of patient recruitment has been elaborated in Figure 1.

Patients were put into one of three groups depending on their canagliflozin dose: Group 1 were on canagliflozin 100 mg/day (1 tablet of CANA-100),
Group 2 were on canagliflozin $150 \mathrm{mg} /$ day (half tablet of CANA-300), and Group 3 were on canagliflozin $300 \mathrm{mg} /$ day (1 tablet of CANA-300).

Patients in Group-2 (canagliflozin $150 \mathrm{mg} / \mathrm{d}$ ) were given a demonstration how to split the CANA-300 tablet. CANA-300 tablet is relatively a big tablet making splitting easier. Using preferably a pill-cutter, the patients were shown how to cut the CANA-300 tablet (Fig. 2A) into 2 halves (Fig. 2B). In case a pill-cutter was not available, the patients were explained that 
a small kitchen knife can also be used to cut the tablet into 2 equal halves. The patients were asked to keep one of the halves in the tablet package carefully to be used the next day (Fig. 2C). They were reassured that sometimes the halves may not be exactly from the middle, but since the patient himself/herself will only be taking the other half of the tablet the subsequent day, it would average out and result in an overall intake of 15 CANA-300 tablets over a period of 30 days.

Data for the following variables were collected at baseline and after 6 months follow-up (height, weight, fasting glucose, 2-hour post prandial glucose and $\mathrm{HbA}_{1 \mathrm{c}}$ ). Additionally, data were collected on haemoglobin, renal function status (creatinine), lipidparameters (low-density lipoprotein cholesterol (LDL-C) and triglycerides) and spot urine albumin creatinine ratio (ACR) as a measure of microvascular complication. Information was noted with regards to occurrence of different adverse drug reactions, specifically, hypoglycaemia, genital infections, complicated upper urinary tract infections, fractures, euglycaemic ketosis and any other event reported by the patients.

\section{Statistical analysis}

Data analysis was done using the Statistical Package for Social Sciences (SPSS) version 20.0 (IBM, USA). Kolmogrov-Smirnov test was used to check the normality of the variable distribution. Normally distributed variables were expressed as mean \pm standard deviation. Skewed variables were expressed as median $\left(25^{\text {th }}-75^{\text {th }}\right.$ percentile). ANOVA was used for comparing three or more study groups. Chi-square test was used for categorical variables. An a priori alpha of $\mathrm{P}<0.05$ was considered statistically significant.

\section{Results}

A total of 3,569 medical records were screened between January 2018 and February 2019, of which 1,232 patients with T2DM were on canagliflozin. Data from 528 patients, who fulfilled all inclusion criteria and for whom at least 6-month follow-up data were available, were analysed. Out of the 528 patients, 257 patients were on canagliflozin $100 \mathrm{mg} /$ day (Group 1; 1 tablet of CANA-100), 138 were on canagliflozin $150 \mathrm{mg} /$ /day (Group 2; half tablet of CANA-300) and 133 were on canagliflozin $300 \mathrm{mg} /$ day (Group 3: 1 tablet of CANA-300). Demographic details, anthropometric, glycaemic, metabolic and medication profiles of the patients in the three groups have been elaborated in Table 1.

The patients in all three groups were comparable with regards to sex distribution, duration of T2DM, baseline $\mathrm{HbA}_{1 \mathrm{c}^{\prime}}$ haemoglobin, renal function (creatinine), lipid parameters and microvasculature damage
(ACR), as per a direct ANOVA of the 3 groups (Table 1) as well as by a post-hoc pair wise analysis between each of the groups (Tables 2-4). The groups were also comparable with regards to use of all the other different anti-diabetes medications (metformin, sulfonylureas, dipeptidyl-peptidase-4 inhibitors, alpha glucosidase inhibitors, pioglitazone, glucagon like peptide-1 receptor agonists and insulins; as per a direct ANOVA of the 3 groups (Table 1) as well as by a post-hoc pair wise analysis between each of the groups (Tables 2-4). However, the patients in Group 2 were significantly younger as compared to patients in Groups 1 and 3 (Tables 1-4). Additionally, BMI was significantly higher in patients in Group 2 as compared to Groups 1 and 3 (Tables 1-4). Patients in Group-3 had significantly higher systolic and diastolic blood pressure (Table 3 ). Patients in Group-2 had a significantly higher diastolic blood pressure, but comparable systolic blood pressure as compared to Group-1 (Table 4).

Following 6 months of treatment, the absolute weight loss was highest in patients receiving canagliflozin $150 \mathrm{mg} /$ day (Group 2; $-3.5 \mathrm{~kg}$ [-6.60 to 0.00]) as compared to those receiving $100 \mathrm{mg} /$ day (Group 1; $-1.05 \mathrm{~kg}$ [-2.85 to -0.17$]$ ) and $300 \mathrm{mg} /$ day (Group 3; $-3.0 \mathrm{~kg}[-5.3$ to -0.8$])$, which was statistically significant $(P=0.002)$ (Table 1$)$. The percent weight loss after 6 months of therapy (which is not effected by the higher baseline BMI) was also significantly higher in Group $2(-3.62 \%)$ as compared to $-3.33 \%$ and $-1.31 \%$ in Groups 3 and Group 1 respectively, which was statistically significant $(P=0.014)$ (Table 1). Post-hoc analysis between each of the 3 groups re-confirmed this observation. A significantly higher absolute and percent weight loss among patients in Group-3 vs Group-1 (Table 3), Group-2 vs Group-1 (Table 4) with comparable absolute and percent weight loss among patients in Group-3 vs Group-2 (Table 2) highlights the superiority of canagliflozin $150 \mathrm{mg} /$ day and 300 $\mathrm{mg} /$ day over canagliflozin $100 \mathrm{mg} /$ day with regards to weight loss.

In terms of glycaemic efficacy, the fall in $\mathrm{HbA}_{1 \mathrm{c}}$ after 6 months of therapy, and the final $\mathrm{HbA}_{1 \mathrm{c}}$ were not statistically different among the three groups $(P=0.083)$ (Table 1). Post-hoc analysis between each of the 3 groups re-confirmed this observation (Tables 2-4). The baseline $\mathrm{HbA}_{1 c^{\prime}}$ the fall in $\mathrm{HbA}_{1 \mathrm{c}}$ after 6 months of therapy and the final $\mathrm{HbA}_{1 \mathrm{c}}$ were comparable when Group-3 was compared to Group-1 (Table 3), Group-2 was compared to Group-1 (Table 4), and when Group-3 was compared to Group-2 (Table 2) Groups 2 and 3 were significantly different only with regards to age and in their baseline BMI (Table 2), hence a separate, post-hoc analysis of the study outcomes was done 
Table 1. Baseline demographics, treatment parameters and outcomes after 6 months of follow-up in patients receiving different graded doses of canagliflozin

\begin{tabular}{|c|c|c|c|c|}
\hline \multirow[t]{2}{*}{ Parameter } & \multicolumn{3}{|c|}{ Canagliflozin study groups } & \multirow[t]{2}{*}{$P$ value } \\
\hline & $\begin{array}{c}\text { Group } 1 \\
\text { Canagliflozin } \\
100 \mathrm{mg} / \text { day } \\
\mathrm{n}=257\end{array}$ & $\begin{array}{c}\text { Group } 2 \\
\text { Canagliflozin } \\
150 \text { mg/day } \\
n=138\end{array}$ & $\begin{array}{c}\text { Group } 3 \\
\text { Canagliflozin } \\
300 \text { mg/day } \\
n=133\end{array}$ & \\
\hline Age, years & $54.26 \pm 10.58$ & $45.31 \pm 13.86$ & $54.62 \pm 10.08$ & $<0.001$ \\
\hline Sex, male:female & 139:118 & $78: 61$ & $71: 62$ & 0.891 \\
\hline Duration of diagnosis, years (range)* & $4.5(2.0-8.0)$ & $4.0(2.0-5.0)$ & $4.0(2.07-7.0)$ & 0.275 \\
\hline BMI at baseline, $\mathrm{kg} / \mathrm{m}^{2}$ & $27.96 \pm 5.29$ & $34.95 \pm 5.76$ & $32.92 \pm 5.78$ & $<0.001$ \\
\hline SBP, mm Hg & $131.12 \pm 19.70$ & $134.92 \pm 21.31$ & $135.89 \pm 19.42$ & 0.057 \\
\hline $\mathrm{DBP}, \mathrm{mm} \mathrm{Hg}$ & $79.19 \pm 10.32$ & $84.05 \pm 10.61$ & $82.25 \pm 10.41$ & $<0.001$ \\
\hline Weight, kg (range) ${ }^{\dagger}$ & $72.95(65.08-82.38)$ & $93.9(80.03-105.98)$ & $84.6(76.6-95.51)$ & $<0.001$ \\
\hline Absolute weight loss at 6 months, $\mathrm{kg}$ (range) ${ }^{\dagger}$ & $-1.05(-2.85$ to -0.17$)$ & $-3.5(-6.60$ to 0.00$)$ & $-3.0(-5.3$ to -0.81$)$ & 0.002 \\
\hline Percent weight loss at 6 months, $\%$ (range) $^{\dagger}$ & $-1.31(-3.28$ to -0.22$)$ & $-3.62(-6.64$ to 0.00$)$ & $-3.33(-6.00$ to -0.99$)$ & 0.014 \\
\hline $\mathrm{HbA}_{1 \mathrm{c}^{\prime}} \%$ (range) & $8.1(7.0-9.6)$ & $8.1(6.8-9.2)$ & $8.8(7.5-9.6)$ & 0.153 \\
\hline $\mathrm{HbA}_{1 \mathrm{c}^{\prime}} \mathrm{mmol} / \mathrm{mol}\left(\right.$ range) ${ }^{\dagger}$ & $65(53-81)$ & $65(51-77)$ & $73(58-81)$ & \\
\hline $\mathrm{HbA}_{1 \mathrm{c}}$ at 6 months, $\%$ (range) & $7.70(6.4-8.6)$ & $7.0(6.1-8.0)$ & $7.2(6.1-8.4)$ & 0.303 \\
\hline $\mathrm{HbA}_{1 \mathrm{c}}$ at 6 months, $\mathrm{mmol} / \mathrm{mol}(\text { range })^{\dagger}$ & $61(46-70)$ & $53(43-64)$ & $55(43-68)$ & \\
\hline 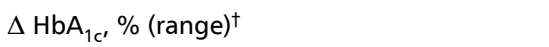 & $-0.75(-2.25$ to 0.15$)$ & $-0.90(-1.83$ to -0.05$)$ & $-0.95(-1.92$ to -0.38$)$ & 0.833 \\
\hline $\mathrm{HbA}_{1 \mathrm{c}}<5.7 \%$ at 6 months, $\mathrm{n}(\%)$ & $6(2.33 \%)$ & $14(10.14 \%)$ & $6(4.51 \%)$ & 0.119 \\
\hline Creatinine, $\mu \mathrm{mol} / \mathrm{L}$ & $78.68 \pm 20.33$ & $69.84 \pm 0.22 .10$ & $78.68 \pm 30.94$ & 0.159 \\
\hline Haemoglobin, gm/dL & $12.08 \pm 1.91$ & $3.23 \pm 1.99$ & $12.51 \pm 2.22$ & \\
\hline LDL-C, mmol/L $\mathrm{L}^{\dagger}$ (range) & $2.56(1.74-3.42)$ & $2.85(2.01-3.94)$ & $2.46(2.06-3.45)$ & 0.070 \\
\hline Triglycerides, mmol/ $\mathrm{L}^{\dagger}$ (range) & $1.93(1.30-2.94)$ & $1.81(1.46-2.59)$ & $2.31(1.38-3.27)$ & 0.339 \\
\hline Hypothyroidism, n (\%) & $26(10.12 \%)$ & $30(21.74 \%)$ & $11(8.27 \%)$ & 0.347 \\
\hline Metformin, n (\%) & $229(89.11 \%)$ & $128(92.75 \%)$ & $127(95.49 \%)$ & 0.097 \\
\hline GLP1a, n (\%) & $28(10.89 \%)$ & $25(18.12 \%)$ & $14(10.53 \%)$ & 0.089 \\
\hline DPP4i, n (\%) & 195 (75.88\%) & $98(71.01 \%)$ & $102(76.69 \%)$ & 0.415 \\
\hline Orlistat, n (\%) & $45(17.51 \%)$ & $30(21.74 \%)$ & $27(20.30 \%)$ & 0.587 \\
\hline Pioglitazone, n (\%) & $112(43.58 \%)$ & $49(35.51 \%)$ & $50(37.59 \%)$ & 0.223 \\
\hline Alpha-glucosidase inhibitors, n (\%) & $29(11.28 \%)$ & $21(15.22 \%)$ & $24(18.05 \%)$ & 0.179 \\
\hline Sulfonylureas, n (\%) & $219(85.21 \%)$ & $108(78.26 \%)$ & $111(83.46 \%)$ & 0.163 \\
\hline Basal insulin, n (\%) & $50(19.46 \%)$ & $28(20.29 \%)$ & $37(27.82 \%)$ & 0.148 \\
\hline Short acting insulin, $n(\%)$ & $28(10.89 \%)$ & $20(14.49 \%)$ & $22(16.54 \%)$ & 0.273 \\
\hline ACR, mg/gm (range) & $64.12(32.42-187.14)$ & $63.11(32.24-212.13)$ & $72.11(16.14-331.43)$ & 0.195 \\
\hline Severe hypoglycaemia, $\mathrm{n}$ & 2 & 1 & 2 & 0.743 \\
\hline Non-severe hypoglycaemia, $\mathrm{n}$ & 15 & 11 & 12 & 0.476 \\
\hline Genital infections, $\mathrm{n}$ & 10 & 5 & 7 & 0.725 \\
\hline
\end{tabular}

Normality of the variable distribution calculated using Kolmogorov-Smirov test; all normally distributed variables expressed as mean \pm standard deviation; discreet variables have been expressed as absolute numbers and percentages; $\mathrm{P}<0.05$ considered statistically significant. ANOVA was used for

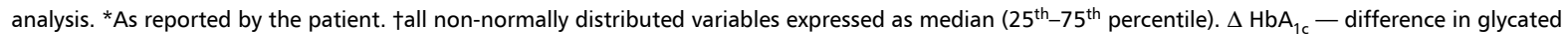
haemoglobin; ACR — spot urine albumin creatinine ratio; BMI — body mass index; DBP — diastolic blood pressure; DPP4i — dipeptidyl peptidase 4 inhibitor; GLP1 a - glucagon like peptide receptor-1 antagonists; $\mathrm{HbA}_{1 \mathrm{c}}$ - glycated haemoglobin; LDL-C — low density lipoprotein cholesterol; SBP — systolic blood pressure

for patients receiving canagliflozin $100 \mathrm{mg} /$ day versus those receiving canagliflozin $150 \mathrm{mg} /$ day or $300 \mathrm{mg} /$ /day (Table 5).

Patients in the post-hoc analysis group (receiving canagliflozin $150 \mathrm{mg} /$ day or $300 \mathrm{mg} /$ day) were signifi- cantly younger, had significantly higher baseline BMI, and had more severe hypertension, but were comparable with regards to use of all the other different anti-diabetes medications (metformin, sulfonylureas, dipeptidyl-peptidase-4 inhibitors, alpha glucosidase 
Table 2. Baseline clinical and treatment parameters and outcomes after 6 months of follow-up in patients receiving canagliflozin $150 \mathrm{mg}$ per day as compared to those receiving $300 \mathrm{mg}$ per day

\begin{tabular}{|c|c|c|c|}
\hline \multirow[t]{5}{*}{ Parameter } & \multicolumn{2}{|c|}{ Canagliflozin study groups } & \multirow[t]{5}{*}{$P$ value } \\
\hline & Group 2 & Group 3 & \\
\hline & Canagliflozin & Canagliflozin & \\
\hline & $150 \mathrm{mg} / \mathrm{d}$ & $300 \mathrm{mg} / \mathrm{d}$ & \\
\hline & $n=138$ & $n=133$ & \\
\hline Age (years) & $45.31 \pm 13.86$ & $54.62 \pm 10.08$ & 0.001 \\
\hline Sex (Male:Female) & $78: 61$ & $71: 62$ & 0.651 \\
\hline Duration of diagnosis (years)* & $4.0[2.0-5.0]$ & $4.0[2.07-7.0]$ & 0.163 \\
\hline BMI $\left[\mathrm{kg} / \mathrm{m}^{2}\right]$ & $34.95 \pm 5.76$ & $32.92 \pm 5.78$ & 0.005 \\
\hline $\mathrm{SBP}[\mathrm{mm} \mathrm{Hg}]$ & $134.92 \pm 21.3$ & $135.89 \pm 19.4$ & 0.707 \\
\hline $\mathrm{DBP}[\mathrm{mm} \mathrm{Hg}]$ & $84.05 \pm 10.61$ & $82.25 \pm 10.4$ & 0.181 \\
\hline Weight $[\mathrm{kg}]^{\dagger}$ & $93.9[80.03-105.98]$ & $84.6[76.6-95.5]$ & 0.001 \\
\hline Weight loss $[\mathrm{kg}]^{\dagger}$ & $-3.5[-6.60-0.00]$ & $-3.0[-5.3$ to -0.8$]$ & 0.813 \\
\hline Percent weight loss at 6 months $(\%)^{\dagger}$ & $-3.62[-6.64-0.00]$ & $-3.33[-6.00$ to -0.99$]$ & 0.734 \\
\hline $\mathrm{HbA}_{1 \mathrm{c}}(\%)$ & $8.1[6.8-9.2]$ & $8.8[7.5-9.6]$ & 0.117 \\
\hline$[\mathrm{mmol} / \mathrm{mol}]^{\dagger}$ & $65[51-77]$ & $73[58-81]$ & 0.335 \\
\hline $\mathrm{HbA}_{1 c}$ at 6 months (\%) & $7.0[6.1-8.0]$ & $7.2[6.1-8.4]$ & \\
\hline$[\mathrm{mmol} / \mathrm{mol}]^{\dagger}$ & $53[43-64]$ & $55[43-68]$ & \\
\hline$\Delta \mathrm{HbA}_{1 \mathrm{c}}(\%)^{\dagger}$ & $-0.90[-1.8$ to -0.05$]$ & $-0.95[-1.92$ to -0.38$]$ & 0.589 \\
\hline $\mathrm{HbA}_{1 \mathrm{c}}<5.7 \%$ at 6 months & $14(10.14 \%)$ & $6(4.5 \%)$ & 0.066 \\
\hline Creatinine $[\mu \mathrm{mol} / \mathrm{L}]$ & $69.84 \pm 0.22 .10$ & $78.68 \pm 30.94$ & 0.113 \\
\hline Haemoglobin $[\mathrm{gm} / \mathrm{dL}]$ & $13.23 \pm 1.99$ & $12.51 \pm 2.22$ & 0.166 \\
\hline $\mathrm{LDL}-\mathrm{C}[\mathrm{mmol} / \mathrm{L}]^{\dagger}$ & $2.85[2.01-3.94]$ & $2.46[2.06-3.45]$ & 0.315 \\
\hline Triglycerides $[\mathrm{mmol} / \mathrm{L}]^{+}$ & $1.81[1.46-2.59]$ & $2.31[1.38-3.27]$ & 0.054 \\
\hline Hypothyroidism & $30(21.73 \%)$ & $11(8.27 \%)$ & 0.246 \\
\hline Metformin & $128(92.75 \%)$ & $127(95.48 \%)$ & 0.247 \\
\hline GLP1a & $25(18.11 \%)$ & $14(10.52 \%)$ & 0.079 \\
\hline DPP4i & $98(71.01 \%)$ & $102(76.69 \%)$ & 0.248 \\
\hline Orlistat & $35(25.36 \%)$ & $27(20.30 \%)$ & 0.338 \\
\hline Pioglitazone & $49(35.50 \%)$ & $50(37.59 \%)$ & 0.688 \\
\hline Alpha-glucosidase inhibitors & $21(15.21 \%)$ & $24(8.04 \%)$ & 0.532 \\
\hline Sulfonylureas & $108(78.26 \%)$ & $111(83.45 \%)$ & 0.231 \\
\hline Basal insulin & $28(20.28 \%)$ & $37(27.81 \%)$ & 0.138 \\
\hline Short acting insulin & $20(14.49 \%)$ & $22(16.5 \%)$ & 0.623 \\
\hline $\mathrm{ACR}[\mathrm{mg} / \mathrm{gm}]$ & $63[32.2-212]$ & $72[16-331]$ & 0.483 \\
\hline
\end{tabular}

Normality of the variable distribution calculated using Kolmogorov Smirov test; All normally distributed variables expressed as mean \pm standard deviation; tall non-normally distributed variables expressed as median [ $25^{\text {th }}-75^{\text {th }}$ percentile]; discreet variables have been expressed as absolute numbers and percents; $\mathrm{P}<0.05$ considered statistically significant; $\Delta \mathrm{HbA}_{1 \mathrm{c}}: \mathrm{HbA}_{1 \mathrm{c}}$ at 6 months $-\mathrm{HbA}_{1 \mathrm{c}}$ at baseline; GLP1a: glucagon like peptide receptor-1 antagonists; BMI: body mass index; DPP4i: dipeptidyl peptidase 4 inhibitor; SBP: systolic blood pressure; DBP: diastolic blood pressure; ACR: spot urine albumin creatinine ratio; LDL-C — low density lipoprotein cholesterol; $\mathrm{HbA}_{1 \mathrm{c}}$ — glycated haemoglobin; *duration of diagnosis (in years) as told by the patient

inhibitors, pioglitazone, glucagon like peptide-1 receptor agonists and insulins) (Table 5). Both absolute and percent weight loss was significantly higher among patients in the post-hoc analysis group (canagliflozin 150 or $300 \mathrm{mg} /$ day) as compared to those receiving $100 \mathrm{mg} /$ day (Table 5). Both basal and final $\mathrm{HbA}_{1 \mathrm{c}}$ after 6 months of therapy were comparable among the groups (Table 5). A greater percent of patients in the post-hoc analysis group (canagliflozin 150 or $300 \mathrm{mg} /$ /day) achieved $\mathrm{HbA}_{1 \mathrm{c}}<5.7 \%$ as compared to those on canagliflozin $100 \mathrm{mg} /$ day but not statistically significant (20 vs $6 ; P=0.428$ ) (Table 5 ).

There were five reports $(0.009 \%)$ of severe hypoglycaemia, necessitating a visit to the hospital emergency department, $38(7.20 \%)$ reports of mild self-limiting hypoglycaemia, 22 reports $(4.17 \%)$ of mild lower 
Table 3. Baseline clinical and treatment parameters and outcomes after 6 months of follow-up in patients receiving canagliflozin $100 \mathrm{mg}$ per day as compared to those receiving $300 \mathrm{mg}$ per day

\begin{tabular}{|c|c|c|c|}
\hline \multirow[t]{5}{*}{ Parameter } & \multicolumn{2}{|c|}{ Canagliflozin study groups } & \multirow[t]{5}{*}{$P$ value } \\
\hline & Group 1 & Group 3 & \\
\hline & Canagliflozin & Canagliflozin & \\
\hline & $100 \mathrm{mg} / \mathrm{d}$ & $300 \mathrm{mg} / \mathrm{d}$ & \\
\hline & $n=257$ & $n=133$ & \\
\hline Age (years) & $54.26 \pm 10.58$ & $54.62 \pm 10.08$ & 0.771 \\
\hline Sex (Male: Female) & 139: 118 & 71: 62 & 0.895 \\
\hline Duration of diagnosis (years)* & $4.5[2.0-8.0]$ & $4.0[2.07-7.0]$ & 0.923 \\
\hline BMI $\left[\mathrm{kg} / \mathrm{m}^{2}\right]$ & $27.96 \pm 5.29$ & $32.92 \pm 5.78$ & $<0.001$ \\
\hline $\mathrm{SBP}[\mathrm{mm} \mathrm{Hg}]$ & $131.12 \pm 19.7$ & $135.89 \pm 19.4$ & 0.027 \\
\hline $\mathrm{DBP}[\mathrm{mm} \mathrm{Hg}]$ & $79.19 \pm 10.32$ & $82.25 \pm 10.4$ & 0.007 \\
\hline Weight $[\mathrm{kg}]^{\dagger}$ & $72.95[65.08-82.38]$ & $84.6[76.6-95.5]$ & $<0.001$ \\
\hline Weight loss $[\mathrm{kg}]^{\dagger}$ & $-1.05[-2.85$ to -0.17$]$ & $-3.0[-5.3$ to -0.8$]$ & $<0.001$ \\
\hline Percent weight loss at 6 months $(\%)^{\dagger}$ & $-1.31[-3.28$ to -0.22$]$ & $-3.33[-6.00$ to -0.99$]$ & 0.002 \\
\hline $\mathrm{HbA}_{1 \mathrm{c}}(\%)$ & $8.1[7.0-9.6]$ & $8.8[7.5-9.6]$ & 0.983 \\
\hline$[\mathrm{mmol} / \mathrm{mol}]^{\dagger}$ & $65[53-81]$ & $73[58-81]$ & \\
\hline $\mathrm{HbA}_{1 \mathrm{c}}$ at 6 months (\%) & $7.70[6.4-8.6]$ & $7.2[6.1-8.4]$ & 0.576 \\
\hline$[\mathrm{mmol} / \mathrm{mol}]^{\dagger}$ & $61[46-70]$ & 55 [43-68] & \\
\hline$\Delta \mathrm{HbA}_{1 \mathrm{c}}(\%)^{\dagger}$ & $-0.75[-2.25-0.15]$ & $-0.95[-1.92$ to -0.38$]$ & 0.627 \\
\hline $\mathrm{HbA}_{1 \mathrm{c}}<5.7 \%$ at 6 months & $6(2.33 \%)$ & $6(4.5 \%)$ & 0.785 \\
\hline Creatinine $[\mu \mathrm{mol} / \mathrm{L}]$ & $78.68 \pm 20.33$ & $78.68 \pm 30.94$ & 0.953 \\
\hline Haemoglobin [gm/dL] & $12.08 \pm 1.91$ & $12.51 \pm 2.22$ & 0.614 \\
\hline LDL-C $[\mathrm{mmol} / \mathrm{L}]^{\dagger}$ & $2.56[1.74-3.42]$ & $2.46[2.06-3.45]$ & 0.304 \\
\hline Triglycerides $[\mathrm{mmol} / \mathrm{L}]^{\dagger}$ & $1.93[1.30-2.94]$ & $2.31[1.38-3.27]$ & 0.629 \\
\hline Hypothyroidism & $26(10.11 \%)$ & $11(8.27 \%)$ & 0.987 \\
\hline Metformin & $229(89.10 \%)$ & $127(95.48 \%)$ & 0.054 \\
\hline GLP1a & $28(10.89 \%)$ & $14(10.52 \%)$ & 0.911 \\
\hline DPP4i & 195 (75.87\%) & $102(76.69 \%)$ & 0.858 \\
\hline Orlistat & $45(17.50 \%)$ & $27(20.30 \%)$ & 0.501 \\
\hline Pioglitazone & $112(43.57 \%)$ & $50(37.59 \%)$ & 0.255 \\
\hline Alpha-glucosidase inhibitors & $29(11.28 \%)$ & $24(8.04 \%)$ & 0.067 \\
\hline Sulfonylureas & $219(85.21 \%)$ & $111(83.45 \%)$ & 0.649 \\
\hline Basal insulin & $50(19.45 \%)$ & $37(27.81 \%)$ & 0.063 \\
\hline Short acting insulin & $28(10.89 \%)$ & $22(16.5 \%)$ & .1170 \\
\hline ACR [mg/gm] & 64 [32-187] & 72 [16-331] & 0.606 \\
\hline
\end{tabular}

Normality of the variable distribution calculated using Kolmogorov Smirov test; All normally distributed variables expressed as mean \pm standard deviation; tall non-normally distributed variables expressed as median [ $25^{\text {th }}-75^{\text {th }}$ percentile]; discreet variables have been expressed as absolute numbers and percents; $\mathrm{P}<0.05$ considered statistically significant; $\Delta \mathrm{HbA}_{1 \mathrm{c}}-\mathrm{HbA}_{1 \mathrm{c}}$ at 6 months- $\mathrm{HbA}_{1 \mathrm{c}}$ at baseline; GLP1a - glucagon like peptide receptor-1 antagonists; $\mathrm{BMI}$ - body mass index; DPP4i - dipeptidyl peptidase 4 inhibitor; SBP — systolic blood pressure; DBP — diastolic blood pressure; ACR - spot urine albumin creatinine ratio; LDL-C — low density lipoprotein cholesterol; $\mathrm{HbA}_{1 \mathrm{c}}$ - glycated haemoglobin; *duration of diagnosis (in years) as told by the patient

genital infection and one report of upper urinary tract infection involving the kidneys. There were no reports of fractures, amputations, euglycaemic ketosis or any hypersensitivity reactions. The occurrence of adverse drug reactions was comparable across the three different dose groups of canagliflozin (Table 1). Monthly cost of canagliflozin $100 \mathrm{mg} / \mathrm{d}, 150 \mathrm{mg} / \mathrm{d}$ and $300 \mathrm{mg} / \mathrm{d}$ was INR 1,635, INR 1,800 and INR 3,600, respectively.

\section{Discussion}

Literature is available to suggest that the function and efficacy of canagliflozin changes with increases in its doses. Polidori et al. [21] reported that the transient intestinal inhibition of SGLT1 was observed with canagliflozin primarily at doses $>200 \mathrm{mg} /$ day. Studies have also suggested that higher doses of canagliflozin have a more sustained 24-hour inhibition of renal glucose 
Table 4. Baseline clinical and treatment parameters and outcomes after 6 months of follow-up in patients receiving canagliflozin $100 \mathrm{mg}$ per day as compared to those receiving $150 \mathrm{mg}$ per day

\begin{tabular}{|c|c|c|c|}
\hline \multirow[t]{5}{*}{ Parameter } & \multicolumn{2}{|c|}{ Canagliflozin study groups } & \multirow[t]{5}{*}{$P$ value } \\
\hline & Group 1 & Group 2 & \\
\hline & Canagliflozin & Canagliflozin & \\
\hline & $100 \mathrm{mg} / \mathrm{d}$ & $150 \mathrm{mg} / \mathrm{d}$ & \\
\hline & $n=257$ & $n=138$ & \\
\hline Age (years) & $54.26 \pm 10.58$ & $45.31 \pm 13.86$ & $<0.001$ \\
\hline Sex (Male: Female) & 139: 118 & $78: 61$ & 0.699 \\
\hline Duration of diagnosis (years)* & $4.5[2.0-8.0]$ & $4.0[2.0-5.0]$ & 0.133 \\
\hline BMI $\left[\mathrm{kg} / \mathrm{m}^{2}\right]$ & $27.96 \pm 5.29$ & $34.95 \pm 5.76$ & $<0.001$ \\
\hline $\mathrm{SBP}[\mathrm{mm} \mathrm{Hg}]$ & $131.12 \pm 19.7$ & $134.92 \pm 21.3$ & 0.096 \\
\hline $\mathrm{DBP}[\mathrm{mm} \mathrm{Hg}]$ & $79.19 \pm 10.32$ & $84.05 \pm 10.61$ & $<0.001$ \\
\hline Weight $[\mathrm{kg}]^{\dagger}$ & $72.95[65.08-82.38]$ & $93.9[80.03-105.98]$ & $<0.001$ \\
\hline Weight loss $[\mathrm{kg}]^{\dagger}$ & $-1.05[-2.85$ to -0.17$]$ & $-3.5[-6.60-0.00]$ & 0.002 \\
\hline Percent weight loss at 6 months $(\%)^{\dagger}$ & $-1.31[-3.28$ to -0.22$]$ & $-3.62[-6.64-0.00]$ & 0.021 \\
\hline $\mathrm{HbA}_{1 \mathrm{c}}(\%)$ & $8.1[7.0-9.6]$ & $8.1[6.8-9.2]$ & 0.118 \\
\hline$[\mathrm{mmol} / \mathrm{mol}] \dagger$ & $65[53-81]$ & $65[51-77]$ & 0.071 \\
\hline $\mathrm{HbA}_{1 \mathrm{c}}$ at 6 months (\%) & $7.70[6.4-8.6]$ & $7.0[6.1-8.0]$ & \\
\hline$[\mathrm{mmol} / \mathrm{mol}]^{\dagger}$ & $61[46-70]$ & $53[43-64]$ & \\
\hline$\Delta \mathrm{HbA}_{1 \mathrm{c}}(\%)^{\dagger}$ & $-0.75[-2.25-0.15]$ & $-0.90[-1.8$ to -0.05$]$ & 0.990 \\
\hline $\mathrm{HbA}_{1 \mathrm{c}}<5.7 \%$ at 6 months & $6(2.33 \%)$ & $14(10.14 \%)$ & 0.135 \\
\hline Creatinine $[\mu \mathrm{mol} / \mathrm{L}]$ & $78.68 \pm 20.33$ & $69.84 \pm 0.22 .10$ & 0.060 \\
\hline Haemoglobin [gm/dL] & $12.08 \pm 1.91$ & $13.23 \pm 1.99$ & 0.011 \\
\hline $\mathrm{LDL}-\mathrm{C}[\mathrm{mmol} / \mathrm{L}]^{\dagger}$ & $2.56[1.74-3.42]$ & $2.85[2.01-3.94]$ & 0.026 \\
\hline Triglycerides $[\mathrm{mmol} / \mathrm{L}]^{\dagger}$ & $1.93[1.30-2.94]$ & $1.81[1.46-2.59]$ & 0.310 \\
\hline Hypothyroidism & $26(10.11 \%)$ & $30(21.73 \%)$ & 0.170 \\
\hline Metformin & $229(89.10 \%)$ & $128(92.75 \%)$ & 0.342 \\
\hline GLP1a & $28(10.89 \%)$ & $25(18.11 \%)$ & 0.063 \\
\hline DPP4i & $195(75.87 \%)$ & $98(71.01 \%)$ & 0.245 \\
\hline Orlistat & $45(17.50 \%)$ & $35(25.36 \%)$ & 0.323 \\
\hline Pioglitazone & $112(43.57 \%)$ & $49(35.50 \%)$ & 0.107 \\
\hline Alpha-glucosidase inhibitors & $29(11.28 \%)$ & $21(15.21 \%)$ & 0.269 \\
\hline Sulfonylureas & $219(85.21 \%)$ & $108(78.26 \%)$ & 0.060 \\
\hline Basal insulin & $50(19.45 \%)$ & $28(20.28 \%)$ & 0.884 \\
\hline Short acting insulin & $28(10.89 \%)$ & $20(14.49 \%)$ & 0.316 \\
\hline ACR [mg/gm] & 64 [32-187] & $63[32.2-212]$ & 0.132 \\
\hline
\end{tabular}

Normality of the variable distribution calculated using Kolmogorov Smirov test; All normally distributed variables expressed as mean \pm standard deviation; tall non-normally distributed variables expressed as median $\left[25^{\text {th }}-75^{\text {th }}\right.$ percentile]; discreet variables have been expressed as absolute numbers and percents; $\mathrm{P}<0.05$ considered statistically significant; $\Delta \mathrm{HbA}_{1 c}-\mathrm{HbA}_{1 c}$ at 6 months- $\mathrm{HbA}_{1 c}$ at baseline; GLP1a - glucagon like peptide receptor-1 antagonists; $\mathrm{BMI}$ - body mass index; DPP4i - dipeptidyl peptidase 4 inhibitor; SBP — systolic blood pressure; DBP — diastolic blood pressure; ACR - spot urine albumin creatinine ratio; LDL-C — low density lipoprotein cholesterol; $\mathrm{HbA}_{1 \mathrm{c}}$ - glycated haemoglobin; ${ }^{*}$ duration of diagnosis (in years) as told by the patient

resorption [22]. A head-to-head pharmacokinetic-pharmacodynamic study of canagliflozin 300 mg versus dapagliflozin $10 \mathrm{mg}$ demonstrated an additional about $25 \%$ lowering of 2-hour prandial glucose with canagliflozin $[22,23]$. Phase III studies of CANA-300 have demonstrated an additional 5-20\% patients achieving $\mathrm{HbA}_{1 \mathrm{c}}<7 \%$ over CANA-100 [23]. Also, CANA-300 was shown to have greater blood-pressure and body-weight lowering trends over CANA-100 in some of the study groups [23]. In a Bayesian network meta-analysis of 13 trials, Shyangdan et al. [24] reported a statistically significant lowering of $\mathrm{HbA}_{1 \mathrm{c}}$ with CANA-300 in monotherapy $(\Delta-0.2 \% ; 95 \%$ confidence interval $[\mathrm{Cl}]-0.05$ to -0.36$)$ and dual therapy as add on to metformin ( $\Delta-0.15 \%$; $95 \% \mathrm{Cl},-0.04$ to -0.26 ), compared to CANA-100. In another larger network meta-analysis 
Table 5. Baseline clinical and treatment parameters and outcomes after 6 months of follow-up in patients receiving canagliflozin $100 \mathrm{mg}$ per day as compared to those receiving $150 \mathrm{mg}$ or $300 \mathrm{mg}$ per day

\begin{tabular}{|c|c|c|c|}
\hline \multirow[t]{5}{*}{ Parameter } & \multicolumn{2}{|c|}{ Canagliflozin study groups } & \multirow[t]{5}{*}{$P$ value } \\
\hline & Group 1 & Group $2+3$ & \\
\hline & Canagliflozin & Canagliflozin & \\
\hline & $100 \mathrm{mg} / \mathrm{d}$ & $150 \mathrm{mg} / \mathrm{d}$ or $300 \mathrm{mg} / \mathrm{d}$ & \\
\hline & $n=257$ & $n=271$ & \\
\hline Age (years) & $54.26 \pm 10.58$ & $49.89 \pm 13.00$ & $<0.001$ \\
\hline Sex (Male:Female) & 139: 118 & $149: 123$ & 0.873 \\
\hline Duration of diagnosis (years)* & $4.5[2.0-8.0]$ & $4.0[2.0-6.0]$ & 0.381 \\
\hline BMI $\left[\mathrm{kg} / \mathrm{m}^{2}\right]$ & $27.96 \pm 5.29$ & $33.91 \pm 5.84$ & $<0.001$ \\
\hline $\mathrm{SBP}[\mathrm{mm} \mathrm{Hg}]$ & $131.12 \pm 19.7$ & $135.4 \pm 20.3$ & 0.018 \\
\hline $\mathrm{DBP}[\mathrm{mm} \mathrm{Hg}]$ & $79.19 \pm 10.32$ & $83.11 \pm 10.52$ & $<0.001$ \\
\hline Weight $[\mathrm{kg}] \dagger$ & $72.95[65.08-82.38]$ & $89.95[79.5-100.07]$ & $<0.001$ \\
\hline Weight loss $[\mathrm{kg}]^{\dagger}$ & $-1.05[-2.85$ to -0.17$]$ & $-3.0[-5.60$ to -0.60$]$ & $<0.001$ \\
\hline Percent weight loss at 6 months $(\%)^{\dagger}$ & $-1.31[-3.28$ to -0.22$]$ & $-3.39[-6.34$ to -0.71$]$ & 0.004 \\
\hline $\mathrm{HbA}_{1 \mathrm{c}}(\%)$ & $8.1[7.0-9.6]$ & $8.4[6.7-9.4]$ & 0.242 \\
\hline$[\mathrm{mmol} / \mathrm{mol}]^{\dagger}$ & $65[53-81]$ & $68[50-79]$ & \\
\hline $\mathrm{HbA}_{1 \mathrm{c}}$ at 6 months (\%) & $7.70[6.4-8.6]$ & $7.1[6.1-8.0]$ & 0.230 \\
\hline$[\mathrm{mmol} / \mathrm{mol}]^{\dagger}$ & $61[46-70]$ & $54[43-64]$ & \\
\hline$\Delta \mathrm{HbA}_{1 \mathrm{c}}(\%)^{\dagger}$ & $-0.75[-2.25-0.15]$ & $-0.9[-1.9$ to -0.3$]$ & 0.769 \\
\hline $\mathrm{HbA}_{1 \mathrm{c}}<5.7 \%$ at 6 months & $6(2.33 \%)$ & $20(7.38 \%)$ & 0.429 \\
\hline Creatinine $[\mu \mathrm{mol} / \mathrm{L}]$ & $78.68 \pm 20.33$ & $74.26 \pm 26.52$ & 0.258 \\
\hline Haemoglobin [gm/dL] & $12.08 \pm 1.91$ & $12.86 \pm 2.3$ & 0.069 \\
\hline $\mathrm{LDL}-\mathrm{C}[\mathrm{mmol} / \mathrm{L}]^{\dagger}$ & $2.56[1.74-3.42]$ & $2.67[2.05-3.73]$ & 0.101 \\
\hline Triglycerides $[\mathrm{mmol} / \mathrm{L}]^{\dagger}$ & $1.93[1.30-2.94]$ & 178 [129-249.5] & 0.756 \\
\hline Hypothyroidism & $26(10.11 \%)$ & $41(15.12 \%)$ & 0.313 \\
\hline Metformin & $229(89.10 \%)$ & $255(94.09 \%)$ & 0.079 \\
\hline GLP1a & $28(10.89 \%)$ & 39 (14.39\%) & 0.234 \\
\hline DPP4i & 195 (75.87\%) & $200(73.8 \%)$ & 0.535 \\
\hline Orlistat & $45(17.50 \%)$ & $57(21.031 \%)$ & 0.186 \\
\hline Pioglitazone & $112(43.57 \%)$ & $99(36.53 \%)$ & 0.098 \\
\hline Alpha-glucosidase inhibitors & $29(11.28 \%)$ & $45(16.61 \%)$ & 0.081 \\
\hline Sulfonylureas & $219(85.21 \%)$ & $219(80.81 \%)$ & 0.152 \\
\hline Basal insulin & $50(19.45 \%)$ & $65(23.98 \%)$ & 0.224 \\
\hline Short acting insulin & $28(10.89 \%)$ & $42(15.49 \%)$ & 0.127 \\
\hline ACR $[\mathrm{mg} / \mathrm{gm}]$ & $64[32-187]$ & $64[32-415]$ & 0.204 \\
\hline
\end{tabular}

Normality of the variable distribution calculated using Kolmogorov Smirov test; All normally distributed variables expressed as mean \pm standard deviation; tall non-normally distributed variables expressed as median [ $25^{\text {th }}-75^{\text {th }}$ percentile]; discreet variables have been expressed as absolute numbers and percents; $\mathrm{P}<0.05$ considered statistically significant; $\Delta \mathrm{HbA}_{1 \mathrm{c}}-\mathrm{HbA}_{1 \mathrm{c}}$ at 6 months- $\mathrm{HbA}_{1 \mathrm{c}}$ at baseline; GLP1a - glucagon like peptide receptor-1 antagonists; $\mathrm{BMI}$ - body mass index; DPP4i - dipeptidyl peptidase 4 inhibitor; SBP — systolic blood pressure; DBP — diastolic blood pressure; ACR — spot urine albumin creatinine ratio; LDL-C — low density lipoprotein cholesterol; $\mathrm{HbA}_{1 \mathrm{c}}$ — glycated haemoglobin; ${ }^{*}$ duration of diagnosis (in years) as told by the patient

of 38 trials involving 23,997 patients, a statistically significant reduction of $\mathrm{HbA}_{1 \mathrm{c}}(\Delta-0.1 \% ; 95 \% \mathrm{Cl}, 0.00$ to -0.20$)$, fasting glucose $(\Delta-0.33 \mathrm{mmol} / \mathrm{L} ; 95 \% \mathrm{Cl}$, -0.07 to -0.90$)$, body weight $(\Delta-0.61 \mathrm{~kg} ; 95 \% \mathrm{Cl}$, -0.23 to -0.99$)$ and SBP $(\Delta-0.98 \mathrm{~mm} \mathrm{Hg} ; 95 \% \mathrm{Cl}, 0.00$ to -1.96$)$ was noted with CANA-300 as compared to CANA-100 [25].
Although there are no head-to-head comparison studies on the efficacy of different SGLT2 inhibitors, Singh et al. [23], through indirect comparison of results of different clinical trials, noted that canagliflozin $300 \mathrm{mg}$ gave the highest reduction in $\mathrm{HbA}_{1 \mathrm{c}}$ (either monotherapy or as a part of multidrug therapy), noting an additional $\mathrm{HbA}_{1 \mathrm{c}}$ lowering of $0.11-0.33 \%$, in 
the background of similar baseline $\mathrm{HbA}_{1 \mathrm{c}}$ and duration of the studies. With regards to weight loss, weight reduction appeared larger with CANA-300 in clinical trials, except when CANA-300 was a part of tripledrug therapy with sulfonylureas and metformin [23]. However, baseline body weights were also different to start with in these patients [23]. In the Bayesian network meta-analysis from 13 trials by Shyangdan et al. (vide supra) CANA-300 was shown to have a greater glycaemic efficacy than other SGLT2 inhibitors as monotherapy (additional $\mathrm{HbA}_{1 \mathrm{c}}$ reduction of $\Delta-0.37 \%$; $95 \% \mathrm{Cl}-0.16$ to -0.58 w.r.t empagliflozin $25 \mathrm{mg} /$ day and $\Delta-0.64 \% ; 95 \% \mathrm{Cl}-0.45$ to -0.83 with regards to dapagliflozin $10 \mathrm{mg} /$ day) [24]. With regards to weight reduction CANA-300 was associated with a statistically significant reduction of weight as compared to empagliflozin $10 \mathrm{mg} /$ day [24]. These results were replicated in a network meta-analysis by Zaccardi et al. (vide supra) [25]. CANA-300 was associated with an additional $0.21 \%$ and $0.20 \%$ lowering of $\mathrm{HbA}_{1 \mathrm{c}}$ w.r.t dapagliflozin $10 \mathrm{mg} /$ day and empagliflozin $25 \mathrm{mg} /$ day, respectively, without any statistically significant difference in body weight reduction. The differences were blunted when SGLT2 inhibitors were used as a part of dual- or multi-drug therapy [23-25]. Hence, preclinical data as well as data from clinical trials suggest that not only canagliflozin may be the most potent SGLT2 inhibitor, because of its additional SGLT1 inhibiting properties, but also that higher doses of canagliflozin may have increased therapeutic benefits.

Our study demonstrated, for the first time in a real-world setting, that higher doses of canagliflozin, $150 \mathrm{mg} /$ day and $300 \mathrm{mg} /$ day, were superior in terms of causing both absolute and percent weight loss as compared to canagliflozin $100 \mathrm{mg} /$ day when used as a part of standard of care for managing diabesity. The highlight of this study is the comparable use of all the different anti-diabetes medications across all the three study groups, especially medications which are linked with mild weight gain like sulfonylureas, pioglitazone and insulin, thus negating any potential impact of these medications on the study outcomes (glycaemic efficacy and weight loss).

It is important to highlight here that the differences in the baseline BMI may have impacted the absolute weight loss, but has no impact on percent weight loss. Following 6 months of treatment, the absolute weight loss was significantly higher in patients receiving canagliflozin $150 \mathrm{mg} /$ day as compared to those receiving $100 \mathrm{mg} /$ day and $300 \mathrm{mg} /$ day $(P=0.002)$ (Table 1$)$. The highest baseline BMI in canagliflozin $150 \mathrm{mg} / \mathrm{d}$ group may have contributed to the greater absolute weight loss in that group. However, it must be realised that the percent weight loss after 6 months of therapy (which is not effected by the higher baseline $\mathrm{BMI}$ ) was also significantly higher in canagliflozin $150 \mathrm{mg} / \mathrm{d}$ group as compared to canagliflozin $300 \mathrm{mg} / \mathrm{d}$ and canagliflozin $100 \mathrm{mg} / \mathrm{d}$ group ( $\mathrm{P}=0.014)$. Post-hoc analysis confirmed that in terms of both absolute and percent weight loss, canagliflozin $150 \mathrm{mg} /$ day and $300 \mathrm{mg} /$ day performed similarly.

The glycaemic efficacy was comparable across the three different doses of canagliflozin used in this study. Since canagliflozin was used as a part of multi drug therapy in this real-world study, this may explain the lack of difference in $\mathrm{HbA}_{1 \mathrm{c}}$ reduction across the different doses of canagliflozin. This study provided reassuring data, for the first time, that the glycaemic and the weight-loss benefits of CANA-300 tablet is retained, even when it is broken into half and taken over 2 different days. "Tablet splitting" not something new, and has been in practice for a long time in India, USA and many other countries across the globe. Freeman et al in a review of PubMed (1966-June 2011) and International Pharmaceutical Abstract (1975-June 2011) found 17 studies dealing with different clinical outcomes, patient acceptance or economic benefits of "tablet splitting" [26]. Patients with chronic disorders, which often needed life-long therapy were most commonly doing "tablet-splitting" viz those on statins, anti-hypertensive medications and anti-psychotics. Their main conclusion was "tablet splitting" did not seem to effect clinical outcomes related to hypertension, cholesterol, or psychiatric disorders [26]. The authors' personal observation are that tablet splitting is commonly practiced in India with regards to diabetes medications, as especially with relatively costlier medications like SGLT2 inhibitors.

Canagliflozin $150 \mathrm{mg} /$ day (half tablet of CANA-300) is significantly cheaper, having a monthly cost of therapy INR 1,800, as compared to INR 3,600 for CANA-300 [13, 14]. The monthly cost of canagliflozin $150 \mathrm{mg} /$ day is only marginally higher than canagliflozin $100 \mathrm{mg} /$ day (INR 1,800 versus INR 1,635, respectively) $[13,14]$, but the therapeutic benefits of canagliflozin $150 \mathrm{mg} /$ day is superior to $100 \mathrm{mg} /$ day.

The limitations of this study include the lack of matching of study groups at baseline, especially with regards to age and body weight. These are limitations intrinsic to real-world studies, where matching and randomisation is not possible. Hence, we have focussed on percent weight loss and not absolute weight loss, which would not be affected by the baseline weight/ /BMI. Other limitations include the short study period of 6 months, making it difficult to assess long-term weight loss, and the lack of robust data on adherence. 
However, as a department policy we always encourage our patients to carry medicines with them whenever they come for visits to the outpatient department (for checking and verification), and collect back empty packs of medicines from patients during these follow-up visits to ensure a good compliance of medication intake. This study highlights the significant cost benefits of using half tablet of CANA-300 in clinical practice, without any compromise in the glycaemic efficacy and weight loss properties of this molecule.

To summarise, this is the first study, to date, that documents the glycaemic efficacy, durability and weight-loss potential of half tablet of CANA-300 taken once a day over a period of 6 months. Half tablet of CANA-300 (150 mg/day) is associated with a significantly greater weight loss and comparable glycaemic efficacy as compared to 1 tablet of CANA-100 with similar costing. Half tablet of CANA-300 (150 mg/day) has glycaemic and weight-loss efficacy equivalent to that of 1 tablet of CANA-300 when used as a part of multi-drug therapy for managing diabesity in India.

\section{Disclosures}

$D D, M S, A D, S A$ and $D K$ have no conflicts of interest, and nothing to declare in relation to this article.

\section{REFERENCES}

1. Dutta $D$, Mukhopadhyay S. Intervening at prediabetes stage is critical to controlling the diabetes epidemic among Asian Indians. Indian J Med Res. 2016; 143(4): 401-404, doi: 10.4103/09715916.184281, indexed in Pubmed: 27377494.

2. Dutta D, Choudhuri S, Mondal SA, et al. Urinary albumin:creatinine ratio predicts prediabetes progression to diabetes and reversal to normoglycemia: role of associated insulin resistance, inflammatory cytokines and low vitamin D. J Diabetes. 2014; 6(4): 316-322, doi: 10.1111/1753-0407.12112, indexed in Pubmed: 24251376.

3. Mondal SA, Dutta D, Kumar M, et al. Neck circumference to height ratio is a reliable predictor of liver stiffness and nonalcoholic fatty liver disease in prediabetes. Indian J Endocrinol Metab. 2018; 22(3): 347-354, doi: 10.4103/ijem.IJEM_31_18, indexed in Pubmed: 30090726.

4. Deepa M, Grace M, Binukumar B, et al. CARRS Surveillance Research Group. High burden of prediabetes and diabetes in three large cities in South Asia: The Center for cArdio-metabolic Risk Reduction in South Asia (CARRS) Study. Diabetes Res Clin Pract. 2015; 110(2): 172-182, doi: 10.1016/j.diabres.2015.09.005, indexed in Pubmed: 26432412.

5. Singla R, Garg A, Singla $S$, et al. Temporal change in profile of association between diabetes, obesity, and age of onset in urban India: a brief report and review of literature. Indian J Endocrinol Metab. 2018; 22(3): 429-432, doi: 10.4103/ijem.IJEM_601_17, indexed in Pubmed: 30090739.

6. Dutta D, Jaisani R, Khandelwal D, et al. Role of metformin, sodium-glucose cotransporter-2 (SGLT2) inhibitors, glucagon-like peptide-1 (GLP-1) receptor agonists, and orlistat based multidrug therapy in glycemic control, weight loss, and euglycemia in diabesity: a real-world experience. Indian J Endocrinol Metab. 2019; 23(4): 460-467, doi: 10.4103/ijem.IJEM_185_19, indexed in Pubmed: 31741907.
7. Lean MEJ, Leslie WS, Barnes AC, et al. Primary care-led weight management for remission of type 2 diabetes (DiRECT): an open-label, cluster-randomised trial. Lancet. 2018; 391(10120): 541-551, doi: 10.1016/S0140-6736(17)33102-1, indexed in Pubmed: 29221645.

8. Singh AK, Unnikrishnan AG, Zargar AH, et al. Evidence-based consensus on positioning of SGLT2i in type 2 diabetes mellitus in Indians. Diabetes Ther. 2019; 10(2): 393-428, doi: 10.1007/ s13300-019-0562-1, indexed in Pubmed: 30706366.

9. Devineni D, Murphy J, Wang SS, et al. Absolute oral bioavailability and pharmacokinetics of canagliflozin: A microdose study in healthy participants. Clin Pharmacol Drug Dev. 2015; 4(4): 295-304, doi: 10.1002/cpdd.162, indexed in Pubmed: 27136910.

10. Singh AK, Singh R. Spotlight on Canagliflozin 300: review of its efficacy and an indirect comparison to other SGLT-2 inhibitors and long-acting GLP-1 receptor agonists. Expert Rev Clin Pharmacol. 2017; 10(6): 633-647, doi: 10.1080/17512433.2017.1318061, indexed in Pubmed: 28393583.

11. Mentock SM, Ng VY, Narayana R, et al. Treatment-seeking behavior and obstacles to treatment compliance in diabetic patients in Mangaluru, India. Diabetes Metab Syndr. 2017; 11 Suppl 2: S617-S622, doi: 10.1016/j.dsx.2017.04.014, indexed in Pubmed: 28465150.

12. Dalvi V, Mekoth N. Patient non-adherence: an interpretative phenomenological analysis. Int J Health Care Qual Assur. 2017; 30(3): 274-284, doi: 10.1108/JJHCQA-03-2016-0033, indexed in Pubmed: 28350217.

13. $1 \mathrm{mg}$. Canagliflozin $100 \mathrm{mg}$ tablet online purchase. https://www. $1 \mathrm{mg}$. com/drugs/invokana-100mg-tablet-173290 (12.01.2020).

14. $1 \mathrm{mg}$. Canagliflozin $300 \mathrm{mg}$ tablet online purchase. https:// www.1 mg.com/drugs/motivyst-tablet-332650 (12.01.2020).

15. Sharma M, Kumar M, Dutta D. Hydroxychloroquine in diabetes and dyslipidaemia: primum non nocere. Diabet Med. 2020; 37(8): 1404 -1405, doi: 10.1111/dme.14144, indexed in Pubmed: 31557353.

16. $1 \mathrm{mg}$. Actrapid online purchase with a valid prescription. https:// www.1 mg.com/drugs/actrapid-hm-100iu-ml-penfill-248417 (12.01.2020).

17. $1 \mathrm{mg}$. Insulatard online purchase with a valid prescription. https:// www.1 mg.com/drugs/insulatard-hm-100iu-ml-penfill-372998 (12.01.2020)

18. $1 \mathrm{mg}$. Humalog online purchase with a valid prescription. https://www.1 mg.com/drugs/humalog-100iu-ml-solution-forinjection-341834 (12.01.2020)

19. $1 \mathrm{mg}$. Lantus online purchase with a valid prescription. https:// www. $1 \mathrm{mg} . c 0 \mathrm{~m} / \mathrm{drugs} / \mathrm{lantus}$-100iu-ml-solution-for-injection-113528 (12.01.2020).

20. Dutta D, Ghosh S. Young-onset diabetes: An Indian perspective. Indian J Med Res. 2019; 149(4): 441-442, doi: 10.4103/ijmr. IJMR_1938_18, indexed in Pubmed: 31411167.

21. Polidori D, Sha S, Mudaliar S, et al. Canagliflozin lowers postprandial glucose and insulin by delaying intestinal glucose absorption in addition to increasing urinary glucose excretion: results of a randomized, placebo-controlled study. Diabetes Care. 2013; 36(8): 2154-2161, doi: 10.2337/dc12-2391, indexed in Pubmed: 23412078.

22. Sha S, Devineni D, Ghosh A, et al. Canagliflozin, a novel inhibitor of sodium glucose co-transporter 2 , dose dependently reduces calculated renal threshold for glucose excretion and increases urinary glucose excretion in healthy subjects. Diabetes Obes Metab. 2011; 13(7): 669-672, doi: 10.1111/j.1463-1326.2011.01406.x, indexed in Pubmed: 21457428.

23. Singh AK, Singh R. Spotlight on Canagliflozin 300: review of its efficacy and an indirect comparison to other SGLT-2 inhibitors and long-acting GLP-1 receptor agonists. Expert Rev Clin Pharmacol. 2017; 10(6): 633-647, doi: 10.1080/17512433.2017.1318061, indexed in Pubmed: 28393583.

24. Shyangdan DS, Uthman OA, Waugh N. SGLT-2 receptor inhibitors for treating patients with type 2 diabetes mellitus: a systematic review and network meta-analysis. BMJ Open. 2016; 6(2): e009417, doi: 10.1136/bmjopen-2015-009417, indexed in Pubmed: 26911584. 
25. Zaccardi F, Webb DR, Htike ZZ, et al. Efficacy and safety of sodium-glucose co-transporter-2 inhibitors in type 2 diabetes mellitus: systematic review and network meta-analysis. Diabetes Obes Metab. 2016; 18(8): 783-794, doi: 10.1111/dom.12670, indexed in Pubmed: 27059700.
26. Freeman MK, White $\mathrm{W}$, Iranikhah $M$. Tablet splitting: a review of the clinical and economic outcomes and patient acceptance. Second of a 2-part series. Part 1 was published in May 2012 (Consult Pharm 2012;27:239-53). Consult Pharm. 2012; 27(6): 421-430, doi: 10.4140/TCP.n.2012.421, indexed in Pubmed: 22698549. 